\title{
Analysis on safe operation of urban gas pipeline
}

\author{
Yun You ${ }^{1, a}$ and Lin $\mathrm{Li}^{2, \mathrm{~b}}$ \\ ${ }^{1}$ Chongqing University of Science and Technology, Chongqing, China \\ ${ }^{2}$ CCTEG Chongqing Engineering Co.,Ltd.Gas engineering design institute, Chongqing, China \\ a Yeppyoyo@hotmail.com, ${ }^{b}$ Stevielee@163.com
}

Keywords: Fault tree, Safe operation, Minimum cut, Probability importance degree.

\begin{abstract}
Fault tree analysis (FTA) is a widely used method in the system safety analysis. In this approach, the logical relationship between accidents may occur and their various reasons is expressed in an arborescence, which is called fault tree analysis. By qualitative and quantitative analysis of the fault tree, the main reason for the accident can be identified. It can provide a reliable basis for determining the security countermeasures in order to achieve the purpose of predicting and preventing accidents. Combining with the survey of Chongqing City gas pipeline, the gas spill fault tree has been established. Do qualitative and quantitative research on the basic reasons for events in the fault tree, calculate the corresponding importance and accident probability, analyze the possible ways to accidents and finally the suggestions as to how the gas system could operate safely is made.
\end{abstract}

\section{Introduction}

The development of city gas industry is one of the important measures to use energy reasonablyand efficiently, protect environment, prevent air pollution, promote production and improve the living conditions of people. With the implementation of many natural gas projects in our country, natural gas has gradually become the main source of city gas. Chongqing Yuchuan Gas Co., Ltd. is a subsidiary of China National Petroleum Corporation. Yu-chuan company mainly engaged in pipeline gas terminal sales, having 112,692 users, of which 116 industrial users, 286 collective users, 1199 commercial users, 111085 resident users and 6 CNG users. Although urban gas brings a lot of convenience to the city residents, it may lead to accident because of manage problems and improperly use. Especially with the extensive application of urban gas in industrial and civil areas, the number and density of underground pipe network are increasing. At the same time, fire caused by corrosion, damage and leakage, and poisoning frequently occur.

Fault Tree Analysis (FTA) is a system safety analysis method which is widely used. In this approach, the logical relationship between accidents may occur and their various reasons is expressed in an arborescence, which is called fault tree analysis. By qualitative and quantitative analysis of the fault tree, the main reason for the accident can be identified. It can provide a reliable basis for determining the security countermeasuresin order to achieve the purpose of predicting and preventing accidents.

\section{Construction of fault tree}

According to Yuchuan company "2011 2014 production anomaly tracking table" , there are 143 gas accidents during 2011 2014, of which 76 gas leakage accidents, accounting for $53.1 \%$. The statistical results show that gas accidents are mostly gas leaks or accidents caused by leakage.

Table1. Yuchuan company gas accident statistics table

\begin{tabular}{c|c|c|c|c|c}
\hline Years & 2011 & 2012 & 2013 & 2014 & Sum \\
\hline Gas accident & 34 & 25 & 36 & 48 & 143 \\
\hline Leakage accident & 21 & 13 & 10 & 32 & 76 \\
\hline Proportion & $61.8 \%$ & $52 \%$ & $28 \%$ & $66.7 \%$ & $53.1 \%$ \\
\hline
\end{tabular}


According to the principle of the top event of fault tree and the operation of natural gas pipeline network of Yuchuan Company, we select the "natural gas leak" as the top event.

Natural gas leakage includes outdoor gas transmission and distribution system leakage (extranet) and indoor gas supply system leakage (intranet). The relationship between them is OR. Figure 1 is to construct the fault tree after repeated analysis of the logical relationship among top events, intermediate events and the basic reasons. Table 1 is the basic events list of fault tree.

\section{Fault tree analysis}

A.Fault Tree qualitative analysis

The main task of fault tree qualitative analysis is to find out all minimal cut sets or minimal path sets of fault tree. Minimum cut set is an integrant set which cause the occurrence of top event.To some extent, the minimal cut sets can represent the level of system risk. When all basic events of a minimal cut setoccur,the top event is bound to happen.In this paper, Boolean algebra method was used to solve the structure function of gas leakage fault tree diagram. The calculation result of all minimal cut sets is as follows.

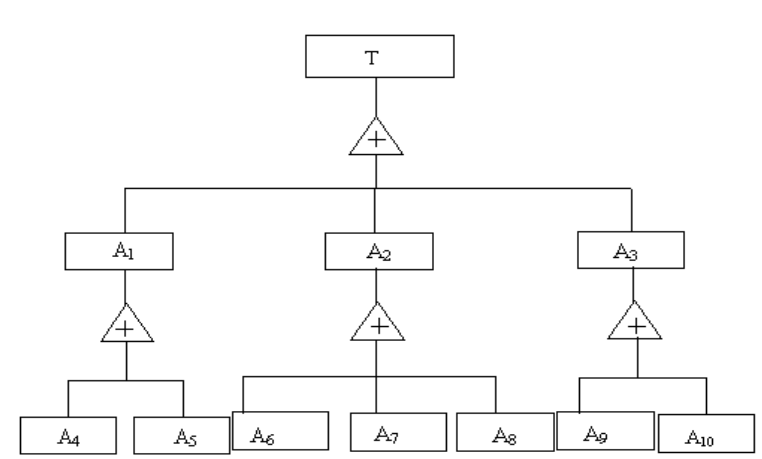

Fig. 1. Analysis diagram of gas leakage fault tree

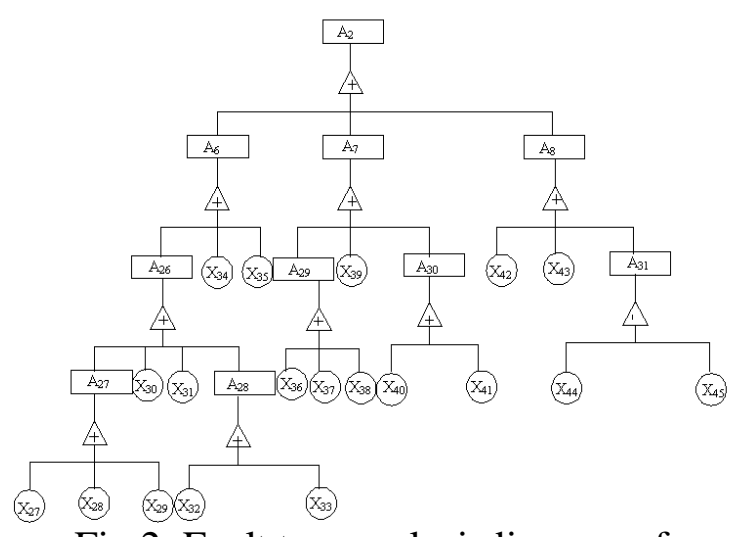

Fig.2. Fault tree analysisdiagram of Pipe network facility leakage

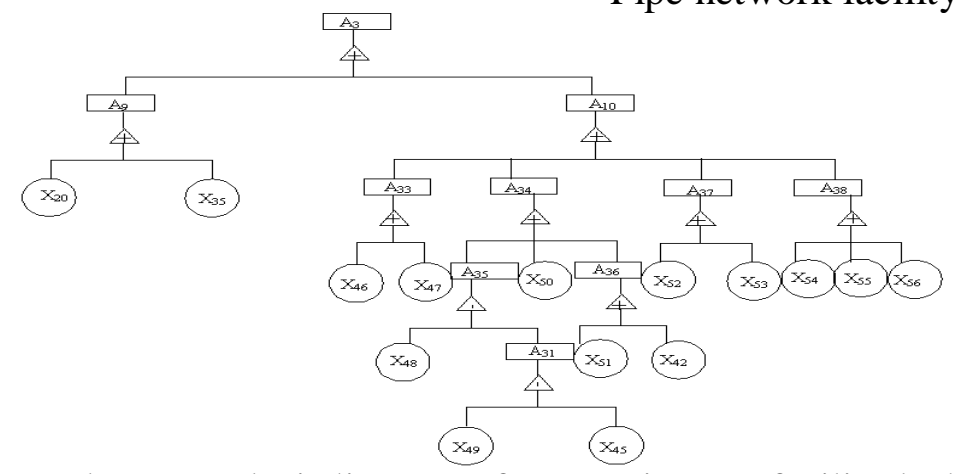

Fig.3. Fault tree analysisdiagram of gas equipment facility leakage

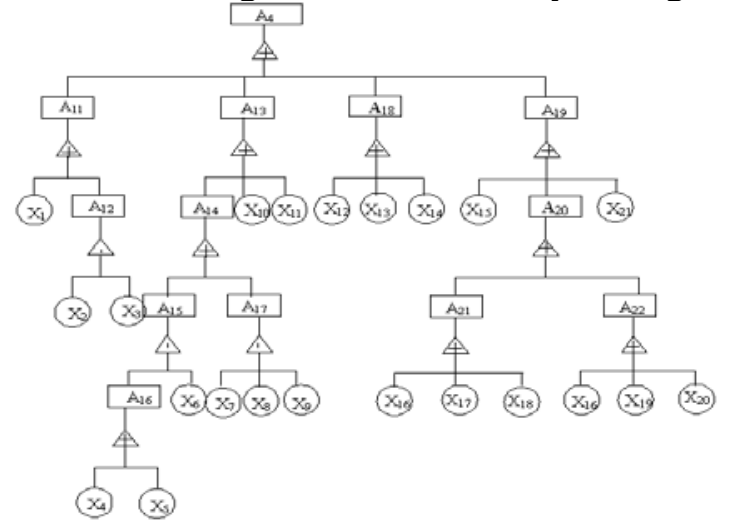

Fig.4. Fault tree analysisdiagram of the external city gas network leakage

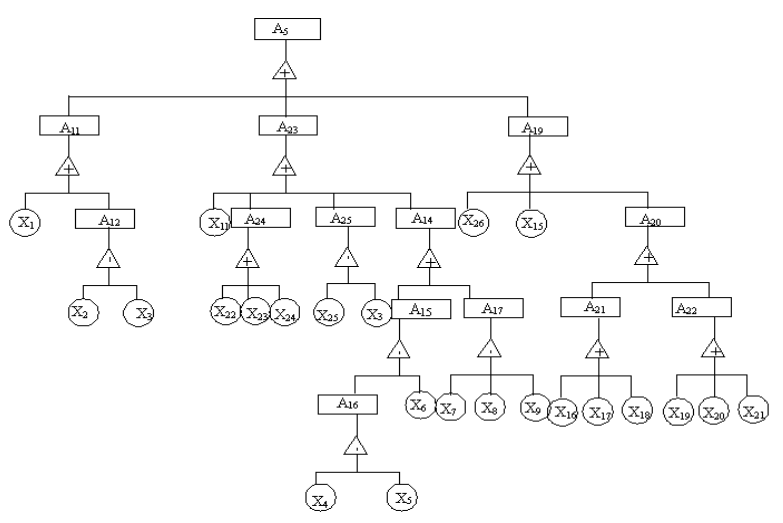

Fig.5. Fault tree analysisdiagram of the internal city gas network leakage 
Table 2. Basic events of fault tree

\begin{tabular}{|c|c|c|c|c|c|c|c|}
\hline Codes & Events & Codes & Events & Codes & Events & $\begin{array}{l}\text { Code } \\
\mathrm{S}\end{array}$ & Events \\
\hline $\mathrm{T}$ & Natural gas leak & $\mathrm{A}_{24}$ & Hot melt defect & $\mathrm{X}_{10}$ & Hot melt defects & $\mathrm{X}_{34}$ & Poor sealing \\
\hline $\mathrm{A}_{1}$ & $\begin{array}{ll}\begin{array}{l}\text { Pipeline } \\
\text { leakage }\end{array} & \text { network } \\
\end{array}$ & $\mathrm{A}_{25}$ & $\begin{array}{ll}\text { Threaded } & \text { connection } \\
\text { leakage } & \\
\end{array}$ & $\mathrm{X}_{11}$ & $\begin{array}{l}\text { Flange connection } \\
\text { leakage }\end{array}$ & $\mathrm{X}_{35}$ & $\begin{array}{l}\text { Third-party } \\
\text { damage }\end{array}$ \\
\hline $\mathrm{A}_{2}$ & Facility leakage & $\mathrm{A}_{26}$ & Poor equipment quality & $\mathrm{X}_{12}$ & $\begin{array}{l}\text { Tube wall thickness } \\
\text { are poorly designed }\end{array}$ & $\mathrm{X}_{36}$ & $\begin{array}{l}\text { Ball valve junction } \\
\text { leakage }\end{array}$ \\
\hline $\mathrm{A}_{3}$ & $\begin{array}{lr}\begin{array}{l}\text { Gas } \\
\text { leakage }\end{array} & \text { equipment } \\
\end{array}$ & $\mathrm{A}_{27}$ & Valve quality problem & $\mathrm{X}_{13}$ & Geological changes & $\mathrm{X}_{37}$ & $\begin{array}{l}\text { Thread leakage at } \\
\text { plug valve }\end{array}$ \\
\hline $\mathrm{A}_{4}$ & $\begin{array}{ll}\begin{array}{l}\text { External } \\
\text { leakage }\end{array} & \text { network } \\
\end{array}$ & $\mathrm{A}_{28}$ & Meter quality problem & $\mathrm{X}_{14}$ & Road over load & $\mathrm{X}_{38}$ & $\begin{array}{l}\text { Other valve } \\
\text { junction leakage }\end{array}$ \\
\hline $\mathrm{A}_{5}$ & $\begin{array}{ll}\begin{array}{l}\text { Internal } \\
\text { leakage }\end{array} & \text { network } \\
\end{array}$ & $\mathrm{A}_{29}$ & $\begin{array}{ll}\begin{array}{l}\text { Valve } \\
\text { leakage }\end{array} & \text { connection } \\
\end{array}$ & $\mathrm{X}_{15}$ & Fatigue cracking & $\mathrm{X}_{39}$ & $\begin{array}{l}\text { Regulator installed } \\
\text { junction leakage }\end{array}$ \\
\hline $\mathrm{A}_{6}$ & Equipment failure & $\mathrm{A}_{30}$ & $\begin{array}{l}\text { Meter } \\
\text { leakage }\end{array}$ & $\mathrm{X}_{16}$ & $\begin{array}{l}\begin{array}{l}\text { Inappropriate } \\
\text { selection } \\
\text { material }\end{array} \\
\end{array}$ & $\mathrm{X}_{40}$ & $\begin{array}{l}\text { Leakage at } \\
\text { connection of flow } \\
\text { meter and pipe }\end{array}$ \\
\hline $\mathrm{A}_{7}$ & $\begin{array}{l}\text { Device junction } \\
\text { leakage }\end{array}$ & $\mathrm{A}_{31}$ & Illegal installation & $\mathrm{X}_{17}$ & $\begin{array}{l}\text { Stray current } \\
\text { interference }\end{array}$ & $\mathrm{X}_{41}$ & $\begin{array}{l}\text { Leakage at } \\
\text { connection of other } \\
\text { instructions and } \\
\text { pipe }\end{array}$ \\
\hline $\mathrm{A}_{8}$ & Illegal operation & $\mathrm{A}_{32}$ & $\begin{array}{ll}\begin{array}{l}\text { Outside } \\
\text { failure }\end{array} & \\
\end{array}$ & $\mathrm{X}_{18}$ & $\begin{array}{l}\text { protecting coating } \\
\text { aging }\end{array}$ & $\mathrm{X}_{42}$ & Operational error \\
\hline $\mathrm{A}_{9}$ & $\begin{array}{l}\text { Anticorrosive } \\
\text { coating failure }\end{array}$ & $\mathrm{A}_{33}$ & $\begin{array}{ll}\text { Appliance quality } \\
\text { problems }\end{array}$ & $\mathrm{X}_{19}$ & $\begin{array}{l}\text { Quality problems of } \\
\text { the installation }\end{array}$ & $\mathrm{X}_{43}$ & Illegal operation \\
\hline $\mathrm{A}_{10}$ & Appliance leakage & $\mathrm{A}_{34}$ & $\begin{array}{l}\text { Management } \\
\text { deficiencies }\end{array}$ & $\mathrm{X}_{20}$ & Poorly designed & $\mathrm{X}_{44}$ & $\begin{array}{l}\text { Installation isn't in } \\
\text { strict accordance } \\
\text { with the procedures }\end{array}$ \\
\hline $\mathrm{A}_{11}$ & Quality problems & $\mathrm{A}_{35}$ & $\begin{array}{l}\text { Improper gas appliance } \\
\text { installation }\end{array}$ & $\mathrm{X}_{21}$ & $\begin{array}{l}\text { Third-party of } \\
\text { external network }\end{array}$ & $\mathrm{X}_{45}$ & $\begin{array}{l}\text { Hasn't checked } \\
\text { after installation }\end{array}$ \\
\hline $\mathrm{A}_{12}$ & $\begin{array}{l}\text { Construction } \\
\text { quality defects }\end{array}$ & $\mathrm{A}_{36}$ & $\begin{array}{l}\text { Weak } \quad \text { security } \\
\text { awareness }\end{array}$ & $\mathrm{X}_{22}$ & $\begin{array}{l}\text { Pipes and fittings } \\
\text { don't fit each other }\end{array}$ & $\mathrm{X}_{46}$ & $\begin{array}{lr}\text { Quality problems } \\
\text { brought ren } \\
\text { extended use of } \\
\text { water heater }\end{array}$ \\
\hline $\mathrm{A}_{13}$ & $\begin{array}{l}\text { External network } \\
\text { connection leakage }\end{array}$ & $\mathrm{A}_{37}$ & Appliance failure & $\mathrm{X}_{23}$ & $\begin{array}{l}\text { Hot melt equipment } \\
\text { is not suitable }\end{array}$ & $\mathrm{X}_{47}$ & The aging of stove \\
\hline $\mathrm{A}_{14}$ & Weld defects & $\mathrm{A}_{38}$ & $\begin{array}{ll}\begin{array}{l}\text { Appliance } \\
\text { leakage }\end{array} & \text { connection } \\
\end{array}$ & $\mathrm{X}_{24}$ & $\begin{array}{l}\text { Partial melting } \\
\text { tumor }\end{array}$ & $\mathrm{X}_{48}$ & $\begin{array}{l}\text { Non-licensed } \\
\text { journeyman }\end{array}$ \\
\hline $\mathrm{A}_{15}$ & $\begin{array}{l}\text { Weld strength does } \\
\text { not meet the } \\
\text { requirements }\end{array}$ & $\mathrm{X}_{1}$ & Pipe quality defect & $\mathrm{X}_{25}$ & Thread defect & $\mathrm{X}_{49}$ & Installation errors \\
\hline $\mathrm{A}_{16}$ & $\begin{array}{lr}\begin{array}{l}\text { Welding } \\
\text { execution }\end{array} & \text { process } \\
\text { meet } & \text { doesn't } \\
\text { requirements } & \text { the } \\
\end{array}$ & $\mathrm{X}_{2}$ & $\begin{array}{l}\text { Nonstandard } \\
\text { construction }\end{array}$ & $\mathrm{X}_{26}$ & $\begin{array}{l}\text { Third-part damage } \\
\text { of internal network }\end{array}$ & $\mathrm{X}_{50}$ & $\begin{array}{l}\text { Inferior products } \\
\text { into the market }\end{array}$ \\
\hline $\mathrm{A}_{17}$ & $\begin{array}{l}\text { Excessive } \\
\text { geometric } \\
\text { imperfections in } \\
\text { welding }\end{array}$ & $\mathrm{X}_{3}$ & $\begin{array}{l}\text { The project hasn't been } \\
\text { examined whether it's } \\
\text { qualified }\end{array}$ & $\mathrm{X}_{27}$ & $\begin{array}{l}\text { Ball valve quality } \\
\text { problems }\end{array}$ & $\mathrm{X}_{51}$ & Valve not closed \\
\hline $\mathrm{A}_{18}$ & Pipe base sink & $\mathrm{X}_{4}$ & $\begin{array}{l}\text { Incorrect heat treatment } \\
\text { process }\end{array}$ & $\mathrm{X}_{28}$ & $\begin{array}{l}\text { Plug valve quality } \\
\text { problems }\end{array}$ & $\mathrm{X}_{52}$ & Water heater failure \\
\hline $\mathrm{A}_{19}$ & Fractureopenings & $\mathrm{X}_{5}$ & $\begin{array}{l}\text { The actual welding } \\
\text { process control is wrong }\end{array}$ & $\mathrm{X}_{29}$ & $\begin{array}{l}\text { Other valve quality } \\
\text { problems }\end{array}$ & $\mathrm{X}_{53}$ & Stove fault \\
\hline $\mathrm{A}_{20}$ & Corrosive opening & $\mathrm{X}_{6}$ & $\begin{array}{l}\text { Strength test is not } \\
\text { found }\end{array}$ & $\mathrm{X}_{30}$ & $\begin{array}{l}\text { Regulator quality } \\
\text { problems }\end{array}$ & $\mathrm{X}_{54}$ & $\begin{array}{lr}\text { Leakage } & \text { at } \\
\text { connection } & \text { of } \\
\text { water heater and } \\
\text { pipe }\end{array}$ \\
\hline $\mathrm{A}_{21}$ & $\begin{array}{l}\text { External } \\
\text { anticorrosion } \\
\text { failure } \\
\end{array}$ & $\mathrm{X}_{7}$ & Welder qualification & $\mathrm{X}_{31}$ & $\begin{array}{l}\text { The gas reservoir } \\
\text { quality problems }\end{array}$ & $\mathrm{X}_{55}$ & $\begin{array}{l}\text { Leakage at } \\
\text { connection of stove } \\
\text { and pipe }\end{array}$ \\
\hline $\mathrm{A}_{22}$ & $\begin{array}{l}\text { Cathode protection } \\
\text { failure }\end{array}$ & $\mathrm{X}_{8}$ & Welding environment & $\mathrm{X}_{32}$ & $\begin{array}{l}\text { Flow meter quality } \\
\text { problem }\end{array}$ & $\mathrm{X}_{56}$ & $\begin{array}{l}\text { Leakage caused } \\
\text { byStove } \\
\text { andpipelineinterfac } \\
\text { eis not strong }\end{array}$ \\
\hline $\mathrm{A}_{23}$ & $\begin{array}{l}\text { Internal network } \\
\text { connection leakage }\end{array}$ & $\mathrm{X}_{9}$ & $\begin{array}{l}\text { Execution of welding } \\
\text { process is wrong }\end{array}$ & $\mathrm{X}_{33}$ & $\begin{array}{l}\text { Quality problems of } \\
\text { other instrument }\end{array}$ & & \\
\hline
\end{tabular}


$\mathrm{E} 1=\{\mathrm{X} 1\} ; \mathrm{E} 2=\{\mathrm{X} 2, \mathrm{X} 3\} ; \mathrm{E} 3=\{\mathrm{X} 4, \mathrm{X} 6\} ; \mathrm{E} 4=\{\mathrm{X} 5, \mathrm{X} 6\} ; \mathrm{E} 5=\{\mathrm{X} 7, \mathrm{X} 8, \mathrm{X} 9\} ; \mathrm{E} 6=\{\mathrm{X} 10\} ; \mathrm{E} 7=\{\mathrm{X} 11\} ;$ $\mathrm{E} 8=\{\mathrm{X} 12\} ; \mathrm{E} 9=\{\mathrm{X} 13\} ; \mathrm{E} 10=\{\mathrm{X} 14\} ; \mathrm{E} 11=\{\mathrm{X} 15\} ; \mathrm{E} 12=\{\mathrm{X} 16\} ; \mathrm{E} 13=\{\mathrm{X} 17\} ; \mathrm{E} 14=\{\mathrm{X} 18\} ; \mathrm{E} 15=\{\mathrm{X} 19\} ;$ $\mathrm{E} 16=\{\mathrm{X} 20\} ; \mathrm{E} 17=\{\mathrm{X} 21\} ; \mathrm{E} 18=\{\mathrm{X} 22\} ; \mathrm{E} 19=\{\mathrm{X} 23\} ; \mathrm{E} 20=\{\mathrm{X} 24\} ; \mathrm{E} 21=\{\mathrm{X} 25, \mathrm{X} 3\} ; \mathrm{E} 22=\{\mathrm{X} 26\} ; \mathrm{E} 23=\{\mathrm{X} 27\} ;$ $\mathrm{E} 24=\{\mathrm{X} 28\} ; \mathrm{E} 25=\{\mathrm{X} 29\} ; \mathrm{E} 26=\{\mathrm{X} 30\} ; \mathrm{E} 27=\{\mathrm{X} 31\} ; \mathrm{E} 28=\{\mathrm{X} 32\} ; \mathrm{E} 29=\{\mathrm{X} 33\} ; \mathrm{E} 30=\{\mathrm{X} 34\} ; \mathrm{E} 31=\{\mathrm{X} 35\} ;$ $\mathrm{E} 32=\{\mathrm{X} 36\} ; \mathrm{E} 33=\{\mathrm{X} 37\} ; \mathrm{E} 34=\{\mathrm{X} 38\} ; \mathrm{E} 35=\{\mathrm{X} 39\} ; \mathrm{E} 36=\{\mathrm{X} 40\} ; \mathrm{E} 37=\{\mathrm{X} 41\} ; \mathrm{E} 38=\{\mathrm{X} 42\} ; \mathrm{E} 39=\{\mathrm{X} 43$, $\mathrm{X} 44, \mathrm{X} 45\} ; \mathrm{E} 40=\{\mathrm{X} 46\} ; \mathrm{E} 41=\{\mathrm{X} 47\} ; \mathrm{E} 42=\{\mathrm{X} 48, \mathrm{X} 49, \mathrm{X} 45\} ; \mathrm{E} 43=\{\mathrm{X} 51\} ; \mathrm{E} 44=\{\mathrm{X} 52\} ; \mathrm{E} 45=\{\mathrm{X} 53\}$; $\mathrm{E} 46=\{\mathrm{X} 54\} ; \mathrm{E} 47=\{\mathrm{X} 55\} ; \mathrm{E} 48=\{\mathrm{X} 56\}$ 。

Generally speaking, the minimum cut sets which include less basic events are easier to happen. $\mathrm{X} 2, \mathrm{X} 4, \mathrm{X} 5$ and X25 were stored in a second-order cut set. X3and X6 were placed in two second-order cut sets. X7, X8, X9, X43, X44, X48 and X49exist in a third-order cut set. X45 exists in two third-order cut sets. The remaining events exist in the first-order cut sets.

According toapproximate discriminant of importance:

$$
I_{i}=\sum_{i=1}^{k_{i}}\left(\frac{1}{2}\right)^{N-1}
$$

In the equation above,ki is the number of cut sets which contain $\mathrm{Xi} ; \mathrm{N}$ is the number of basic events in the cut set which contains Xi.

From the above, the structure importance of basic events which exists in first-order cut sets is biggest, X3 and X6 followed by.The importance of X2,X4,X5,X25 and X49 is relatively small. The structure importance of X7, X8, X9, X43, X44, X48 and X49which exists in third-order cut sets is smallest. The resulting order of importance is as follows:

$$
\begin{aligned}
& I_{1}=I_{10}=\ldots=I_{24}=I_{27}=\ldots=I_{43}=I_{47}=I_{50}=\ldots=I_{56}>I_{3}=I_{6}>I_{2}=I_{4}=I_{5}=I_{25}=I_{49}> \\
& I_{7}=I_{8}=I_{9}=I_{43}=I_{44}=I_{48}=I_{49}
\end{aligned}
$$

Therefore, pipe defects, leakage at connection of stove and pipeline and quality defects of facilitiesoccupy relatively important positions and they are the dominant factors whether the top events would happen.

B.Quantitative analysis of the fault tree

The main task of fault tree quantitative analysis is to calculate the probability of occurrences of top events. In quantitative probability calculation, assume that the bottom events are independent and the bottom events have only two conditions, which is normal or accidental condition.

At time $t$, the probability of occurrence of the minimum cut set $\mathrm{j}$ is:

$$
P\left[k_{j}(t)\right]=P\left[\bigcap_{i \in k_{j}} x_{i}(t)\right]=\prod_{i \in k_{j}} F_{i}(t)
$$

In the formula above, $x i$ is the bottom event of minimum cut set kj;Fi(t) is the probability of xi, which is the failure rate of component $\mathrm{i}$.

The probability of occurrence of top event:

$$
P(T)=\bigcup_{J=1}^{N} p\left[k_{j}(t)\right]
$$

In the formula above, $\mathrm{N}$ is the number of minimum cut sets in the system. Bu statistics and analysis of gas accidents in Yuchuan Company in the past few years, the probabilities of occurrence of each basic event can be learned. They are shown as Table 3. 
Table 3. Probability of basic events in gas system

\begin{tabular}{|c|c|c|c|c|c|}
\hline Event & Probability & Event & Probability & Event & Probability \\
\hline $\begin{array}{lr}\text { Leakage } & \text { caused by } \\
\text { pipeline } & \text { facility } \\
\text { corrosion } & \\
\end{array}$ & 0.0001 & Poorly designed & 0.0003 & $\begin{array}{l}\text { Leakage at screw } \\
\text { thread of cocks }\end{array}$ & 0.0065 \\
\hline $\begin{array}{l}\text { Leakage at pipeline } \\
\text { welds }\end{array}$ & 0.0002 & Geological changes & 0.0001 & $\begin{array}{l}\text { The project hasn't } \\
\text { been checked whether } \\
\text { it's qualified }\end{array}$ & 0.0060 \\
\hline $\begin{array}{l}\text { Third-party damage } \\
\text { of external network }\end{array}$ & 0.0003 & Road over load & 0.0003 & Tank leakage & 0.0003 \\
\hline $\begin{array}{l}\text { Leakage caused by } \\
\text { nonstandard } \\
\text { installation of valve }\end{array}$ & 0.0001 & $\begin{array}{l}\text { Inappropriate } \\
\text { selection of material }\end{array}$ & 0.0001 & $\begin{array}{l}\begin{array}{l}\text { Leakage caused by } \\
\text { poor } \\
\text { performance }\end{array} \\
\end{array}$ & 0.0001 \\
\hline $\begin{array}{l}\text { Leakage caused by } \\
\text { quality defects of } \\
\text { pressure regulator }\end{array}$ & 0.0003 & $\begin{array}{l}\text { Leakage caused by } \\
\text { fatigue cracking }\end{array}$ & 0.0003 & $\begin{array}{l}\text { Leakage caused by } \\
\text { insufficient attachment } \\
\text { strength }\end{array}$ & 0.0001 \\
\hline $\begin{array}{l}\text { Leakage caused by } \\
\text { pipecorrosion }\end{array}$ & 0.0002 & $\begin{array}{l}\text { Leakage caused by } \\
\text { quality defects of pipe } \\
\text { material }\end{array}$ & 0.0003 & $\begin{array}{l}\text { Leakage caused by } \\
\text { poor seal }\end{array}$ & 0.0005 \\
\hline $\begin{array}{l}\begin{array}{l}\text { Leakage caused by } \\
\text { aging hose }\end{array} \\
\end{array}$ & 0.0052 & $\begin{array}{l}\begin{array}{l}\text { Leakage at flange } \\
\text { joint }\end{array} \\
\end{array}$ & 0.0003 & $\begin{array}{l}\text { Explosion caused by } \\
\text { welding fire }\end{array}$ & 0.0001 \\
\hline Leakage at weld & 0.0018 & $\begin{array}{l}\text { Leakage caused by } \\
\text { valve quality defects }\end{array}$ & 0.0013 & $\begin{array}{l}\text { Leakage at pressure } \\
\text { regulator }\end{array}$ & 0.0006 \\
\hline $\begin{array}{l}\text { Leakage caused by } \\
\text { flow meter quality } \\
\text { defects }\end{array}$ & 0.0002 & Leakage at valve joint & 0.0020 & $\begin{array}{l}\text { Leakage at pressure } \\
\text { regulator junction }\end{array}$ & 0.0008 \\
\hline $\begin{array}{l}\text { Leakage caused by } \\
\text { aging stove }\end{array}$ & 0.0055 & $\begin{array}{l}\text { Leakage caused by } \\
\text { hose damage }\end{array}$ & 0.0009 & $\begin{array}{|lll|}\begin{array}{l}\text { Leakage } \\
\text { junction }\end{array} & \text { at hose } \\
\end{array}$ & 0.0035 \\
\hline $\begin{array}{l}\text { Leakage at } \\
\text { connection of water } \\
\text { heater and pipe }\end{array}$ & 0.0093 & $\begin{array}{l}\text { Leakage at connection } \\
\text { of screw thread }\end{array}$ & 0.0032 & $\begin{array}{l}\text { Leakage caused by } \\
\text { stove defects }\end{array}$ & 0.0024 \\
\hline $\begin{array}{l}\text { Installation staff is } \\
\text { not eligible }\end{array}$ & 0.0010 & $\begin{array}{l}\text { Leakage at connection } \\
\text { of flow meter and } \\
\text { pipeline }\end{array}$ & 0.0108 & $\begin{array}{l}\text { Leakage caused by } \\
\text { extended use of water } \\
\text { heater }\end{array}$ & 0.0074 \\
\hline $\begin{array}{l}\text { Hasn't checked after } \\
\text { installation }\end{array}$ & 0.0003 & $\begin{array}{l}\text { Leakage at connection } \\
\text { of stove and pipeline }\end{array}$ & 0.0054 & $\begin{array}{l}\text { Faults in stove } \\
\text { installation process }\end{array}$ & 0.0017 \\
\hline $\begin{array}{l}\text { Leakage caused by } \\
\text { cock quality defects }\end{array}$ & 0.0073 & $\begin{array}{l}\text { Leakage caused by } \\
\text { gas appliance quality } \\
\text { defect }\end{array}$ & 0.0010 & $\begin{array}{l}\text { Improperly closed } \\
\text { valves }\end{array}$ & 0.0107 \\
\hline $\begin{array}{l}\text { Staff fail to work in } \\
\text { accordance } \\
\text { procedures }\end{array}$ & 0.0010 & $\begin{array}{l}\text { Leakage caused by } \\
\text { water heater failure }\end{array}$ & 0.0009 & $\begin{array}{lll}\text { Leakage caused by } \\
\text { third-part damage } \\
\text { internal network }\end{array}$ & 0.0063 \\
\hline $\begin{array}{l}\text { Non-standard } \\
\text { construction }\end{array}$ & 0.0010 & $\begin{array}{l}\text { The installation } \\
\text { process is not in strict } \\
\text { accordance with } \\
\text { procedures }\end{array}$ & 0.0052 & $\begin{array}{l}\text { Leakage caused by } \\
\text { pipeline quality defects }\end{array}$ & 0.0001 \\
\hline $\begin{array}{lr}\text { Leakage } & \text { caused by } \\
\text { incorrect } & \text { heat } \\
\text { treatment } & \\
\end{array}$ & 0.0001 & $\begin{array}{l}\text { Leakage caused by } \\
\text { ball valve quality } \\
\text { defects }\end{array}$ & 0.0079 & $\begin{array}{l}\text { Instruments } \\
\text { defects }\end{array}$ & 0.0003 \\
\hline Hot melt defects & 0.0001 & $\begin{array}{l}\text { Leakage at ball valve } \\
\text { joint }\end{array}$ & 0.0065 & $\begin{array}{l}\text { Leakage at instrument } \\
\text { junction }\end{array}$ & 0.0005 \\
\hline
\end{tabular}

Inserting the corresponding probabilities of basic events, the probability of natural gas leakage while running in Yuchuan Company can be calculated:

$$
P(T)=\bigcup_{J=1}^{48} p\left[k_{j}(t)\right]=0.0965
$$

According to the importance analysis, pipe defects, gas appliance failure and damaged pipe fittings are the main reasons for gas leakage. Corresponding probabilities of basic events can be got after further analysis.It is shown as Table 4. 
Table 4. Probabilities of main intermediate events

\begin{tabular}{c|l|c|c|c|c}
\hline Symbol & \multicolumn{1}{|c|}{ Event } & Probability & Symbol & Event & Probability \\
\hline$P\left(A_{2}\right)$ & $\begin{array}{l}\text { Probability of pipe network } \\
\text { facility leakage }\end{array}$ & 0.0380 & $P\left(A_{4}\right)$ & $\begin{array}{l}\text { Probability of leakage at external } \\
\text { city gas network }\end{array}$ & 0.0024 \\
\hline$P\left(A_{3}\right)$ & $\begin{array}{l}\text { Probability of leakage at gas } \\
\text { appliance }\end{array}$ & 0.5090 & $P\left(A_{5}\right)$ & $\begin{array}{l}\text { Probability of leakage at internal } \\
\text { city gas network }\end{array}$ & 0.0066 \\
\hline
\end{tabular}

As can be seen from the table above, leakage at gas appliance is the most important factor for gas leakage while gas system is running. Leakage caused by facilities also cannot be neglected. However, leakage at city gas network is relatively small. In other words, these events should serve as the main basis for accident prevention. Gas company should pay close attention to these factors in order to ensure safety.

\section{Conclusion}

Based on fault treeanalysis, in order to prevent fire and explosion caused by natural gas leakage and improve the level of safe operation of natural gas network in Yuchuan Company, several suggestions are proposed as follows:

(1) Improve the construction of emergency organization system. It is recommended to build a rescue organization hierarchically and by region according to the current emergency rescue and protection work leading group, to bear the scope of their respective jurisdictions.

(2) Improve the emergency response management mechanism. Yuchuan company has established a contingency management system. It is suggested that Yuchuan Company will further improve the rules and regulations, such as plans, systems and methods, and standardize the operation procedures and standards. Monthly inform the gas rescue rate, pipe network leaks self-examination rate, hidden trouble rectification and so on, and sum up lessons.

(3) Improve the information construction of gas pipeline network. Transform urban natural gas pipeline network by new technology such as GIS and SCADA. Establish urban gas pipeline network information system and computer-aided scheduling system, so as to improve the safe operation of natural gas levels.

(4) Strengthen the construction and training of rescue personnel. It is suggested that the company should focus on strengthening the knowledge level of the relevant personnel in the rescue skills, safety awareness, organization and coordination, professional level, actual combat experience and other aspects.

\section{Acknowledgement}

In this paper, the research was sponsored by Science and technology research Project of Chongqing Education Commission (Project No. KJ1501337) and Key project Fund Project of Chongqing University of Science and Technology (Project No.CK2016Z22).

\section{References}

[1] State administration of work safety. Safety Assessment(The third edition) [M].Beijing: Coal Industry Press, 2004

[2] PengShini.Gas safty technology[M].Chongqing:Chongqing University Press， 2005

[3] Zhang Puren.Identificationofhazardous sources and simulation analysis if hazards for gas fire and explosion[J].natural gas industry, 2005, 25(1): 151-154

[4] Liu Yashi. The common faults and treatments in operation of Low-pressure city gas pipeline network[J]. Journal of Henan Urban Construction College, 2002,1(11):14-16 